\title{
TRAFFIC MODELING AND TRACKING FOR MULTIUSER DETECTION FOR RANDOM ACCESS NETWORKS
}

\author{
Biao Chen and Lang Tong \\ School of Electrical Engineering \\ Cornell University, Ithaca, NY 14853 \\ Email: biao[ltong]@ee.cornell.edu
}

\begin{abstract}
Traffic burstiness results in the predictability of user activity at the individual source level, and the exploitation of such predictability in the receiver design for wireless packet switching random access/CDMA networks is investigated in this paper. It is shown that the conventional approach of assuming all users are active results in substantial performance loss when a linear multiuser detector, and in particular, a decorrelating detector is implemented. A two-stage receiver is proposed where the first stage tracks active users and prior to the second stage symbol detection. Two different user trackers are presented in this paper and it is demonstrated that, with the help of traffic predictability, accurate estimate of active set of users is possible, even with a simple matched filter bank implementation at the first stage.
\end{abstract}

\section{INTRODUCTION}

While traffic modeling for packet switching networks has been studied for more than a decade, its usage was limited largely to network performance analysis. With the advent of wireless packet switching networks, however, traffic modeling could be valuable in the receiver design as well. Consider, for example, the uplink of a random access/CDMA packet switching network [7]. Assume transmitter code, i.e., each user encodes its packets with its own spreading code. Each user freely accesses the network whenever it has new packets arriving, therefore the knowledge of active users is not known a priori to the receiver. This random access mechanism is in contrast to most cellular systems where active users at any time is known to the receiver through initial handshaking. The lack of such information prompts two different approaches in the receiver design. The first one, referred to as the conventional approach in the sequel, assumes that all potential users are active. The second one identifies the set of active users first before symbol detection.

This work was supported in part by the National Science Foundation under Contract CCR-9804019 and by the Office of Naval Research under Contract N00014-96-1-0895.
With simple matched filter receivers, the approach of assuming all users are active does not render any performance loss. However, if a multiuser detector (MUD), e.g., a decorrelating detector (DD), is employed to mitigate multiple access interference (MAI) and near far effect, this conventional viewpoint will incur significant performance degradation. It would therefore be better off for the receiver to adopt the second approach, performing user identification prior to symbol detection. The problem of detecting active users has been addressed by several authors in a dynamic CDMA system [5, 4, 9]. A common feature of their approaches is that the detection is focused on current data window without utilizing the traffic continuity. For packet switching networks, however, the traffic burstiness causes highly correlated user activities which results in enhanced predictability of user traffic at the individual source level. Exploitation of such predictability to improve the receiver performance is the topic of this paper and in particular, we adopt a first order approximation of bursty traffic originally proposed by Viterbi in 1986 [8]. The key to utilizing the traffic model is to convert the user identification problem to model selection problem for which the traffic continuity is incorporated in the regressor selection procedure.

The organization of the paper is as follows. In the next section, the signal model is presented, followed by a detailed study of the overmodeling effect on the receiver performance. In section 3, a two stage MUD is proposed where the first stage aims to detect the set of active users. This is followed by some simulation result in section 4 along with some concluding remarks.

\section{OVERMODELING EFFECT}

Consider a slotted random access packet switching system with $M$ subscribers, as in [7]. Each user is assigned a unique spreading code and transmits synchronously over a common channel. Assume BPSK for simplicity, the received signal within a packet slot could be written as

$$
\mathbf{y}_{n}(t)=\sum_{k \in \mathcal{I}_{n}} A^{(k)} b_{n}^{(k)}(t) \mathbf{c}^{(k)}+\mathbf{z}_{n}(t), \quad t=1, \ldots, T
$$


where $n$ is the packet index, $t$ is the symbol index within a packet, $T$ is the packet size, $k$ is the user index, $A^{(k)}, \mathbf{c}^{(k)}$ are the amplitude and the signature vector of user $k$ present at the receiver, $b_{n}^{(k)}(t)$ is the $t^{t h}$ symbol within the $n^{t h}$ packet for user $k, \mathbf{z}_{n}(t)$ is additive white Gaussian noise with zero mean and covariance matrix $\sigma^{2} \mathbf{I}$, and $\mathcal{I}_{n}$ is the index set of the active users at slot $n$, thus $\mathcal{I}_{n} \subset\{1,2, \ldots, M\}$. The receiver's task is to detect the symbols $b_{n}^{(k)}$ for all $k \in \mathcal{I}_{n}$ without prior knowledge of $\mathcal{I}_{n}$.

A linear DD can be viewed as a least square (LS) estimator followed by a sign detector. A direct result of including inactive users in the system - so called overmodeling, is the increased variance of those parameters of interest. To illustrate this, assume only one user is active whose signature vector is $\mathbf{c}$, while $\mathbf{D}$ is the signature vectors of those inactive users erroneously included in the model. It can be shown that the variance of LS solution is the inverse of the magnitude square of $\mathbf{c}$ for the true model; while for the full model it is the inverse of the magnitude square of the projection error $\left[\mathbf{I}-\mathbf{D}\left(\mathbf{D}^{\prime} \mathbf{D}\right)^{-1} \mathbf{D}^{\prime}\right] \mathbf{c}$ (cf. Figure 1). Clearly, the former is always smaller than latter. General result holds when $\mathbf{C}$ has more than one columns (more than one active users) [1].

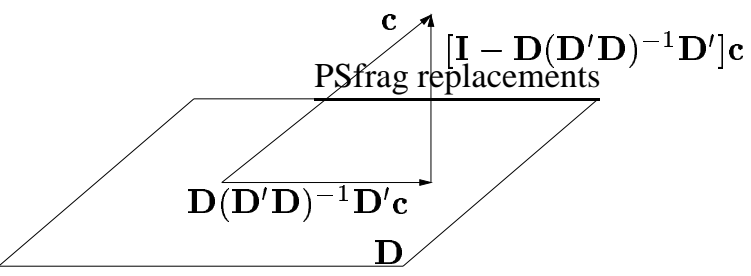

Figure 1: Illustration of the covariance of LS estimate using true and full model.

The increased variance of LS estimate of assuming the full model will increase the symbol detection error probability, which, in turn, degrade the packet success probability (PSP). Notice PSP is also a function of channel traffic condition. Conceptually, if on the average there is only a small number of users being active, then the advantage of correct modeling should be more significant than an overloaded channel. Assume a homogeneous channel traffic with each user at any slot transmitting a packet with probability $q$, hence the overall number of packets (or equivalently, number of active users, denoted by $L$ ) per slot is binomial with success probability $q$. We can obtain packet error rate $(1-P S P)$ as a function of $q$ as shown in Figure 2. The gain in terms of packet error rate is of several magnitude over that of the full model. Also plotted in dashed line is the packet error rate using a matched filter (MF) bank for all users. Clearly, MF receiver is arbitrarily close to the DD using the true model as channel load approaches zero.

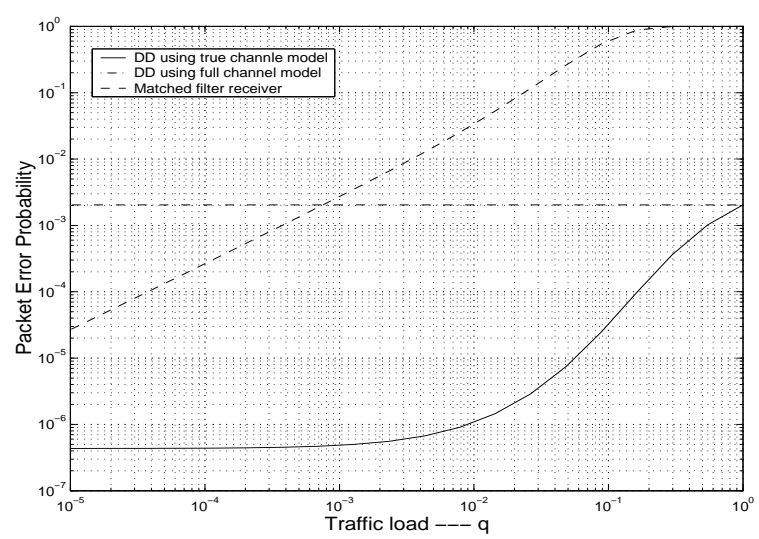

Figure 2: Packet error probability as a function of traffic load $q$ for the decorrelator using full model (dash-dotted line), true model (solid line), and simple matched filter bank (dashed line). The total number of potential users is 30 and the correlation coefficient is assumed to be $\rho=0.2$ for each pair of signature vectors.

\section{TRAFFIC-AIDED MUD}

\subsection{Traffic modeling for packet switching networks}

The performance degradation of a DD when inactive users' signature vectors are erroneously included provokes the idea of a two stage receiver. In the first stage, the set of active users is detected while in the second stage a MUD is implemented by adapting to the change of user profile. To facilitate the user identification, we first introduce the following signal model as an alternative to that of (1).

$$
\mathbf{y}_{n}(t)=\sum_{k=1}^{M} \mathbf{c}^{(k)} s_{n}^{(k)}(t) \gamma_{n}^{(k)}+\mathbf{z}_{n}(t)
$$

where, in the $n$th slot, $\gamma_{n}^{(k)}$ is the indicator function that takes value 1 if the $k^{\text {th }}$ user is active and 0 otherwise, and $s_{n}^{(k)}=A^{(k)} b_{n}^{(k)}$. Incorporating the indicator functions in the signal model provides a natural framework to include the traffic information which will be interpreted as the probability law that dictates the binary process $\gamma_{n}^{(k)}$.

A first order approximation of the bursty traffic for individual source is a two state Markov chain [8]. This Markovian assumption results in geometrical distribution of both the message length (number of contiguous packets) and idle period lengths, which is consistent with the bursty model adopted in [3]. Define $\Gamma_{n}=\left(\gamma_{n}^{(1)}, \ldots, \gamma_{n}^{(M)}\right)$ as the state variable of user profile, i.e., those coordinates that equal to ' 1 ' in $\Gamma_{n}$ indicate that the corresponding users are transmitting at slot $n$. The combined state variable $\Gamma_{n}$ is now also a Markov sequence with state dimensionality equal to $2^{M}$ where $M$ is the total number of potential users. Its transition matrix is simply the Kronecker product of all the transition matrices for each individual $\gamma_{n}^{(k)}$. In turn, our observation 
is now a hidden Markov model (HMM).

$$
\mathbf{y}_{n} \triangleq\left[\begin{array}{c}
\mathbf{y}_{n}(1) \\
\vdots \\
\mathbf{y}_{n}(T)
\end{array}\right]=\sum_{k \in \mathcal{I}\left(\Gamma_{n}\right)}\left[\begin{array}{c}
\mathbf{c}^{(k)} s_{n}^{(k)}(1) \\
\vdots \\
\mathbf{c}^{(k)} s_{n}^{(k)}(T)
\end{array}\right]+\left[\begin{array}{c}
\mathbf{z}_{n}(1) \\
\vdots \\
\mathbf{z}_{n}(T)
\end{array}\right]
$$

where $T$ is the packet size. The detection of active users amounts to the inference of the underlying state $\Gamma_{n}$. The optimal state estimator maximizes the posterior probability, $P\left(\Gamma_{n} \mid \mathbf{y}_{n}, \cdots, \mathbf{y}_{1}\right)$, and this could be evaluated using the so-called forward variable, which admits efficient recursive computation [6]. However, the state dimensionality of $\Gamma_{n}$ is $2^{M}$, meaning there are total number of $2^{M}$ models needs to be evaluated, a usually formidable task in practice.

\subsection{Traffic tracking}

The prohibitive complexity of the optimal HMM tracker motivates alternative (hence non-optimal) approaches whose complexity grow linearly in the number of users. The key is the 'decoupling' of individual user's state tracking, which results in a suboptimal structure shown in Figure 3(a). Specifically, the received signal is first mapped to a new statis-

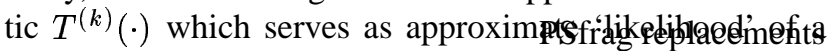
particular user being active given current slot observations. Then, the state of the user's previous slot is used to jointly decide whether the user is transmitting at the current slot.

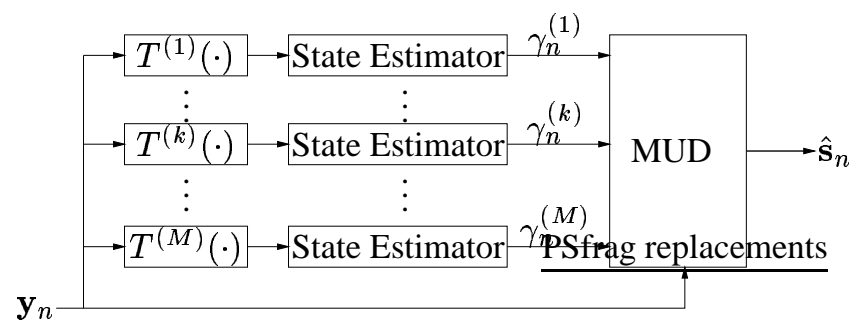

(a) PSfrag replacements

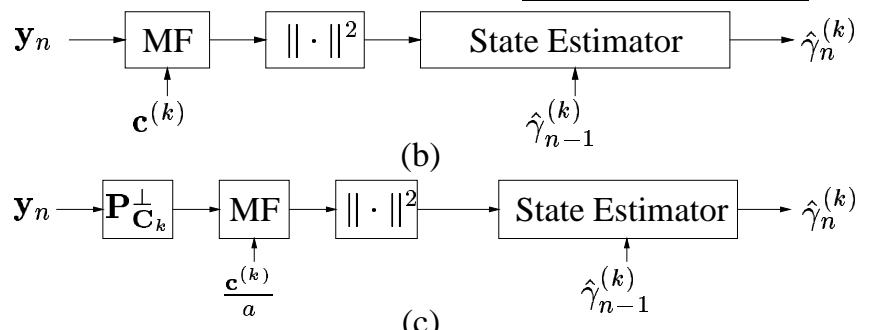

(c)

Figure 3: (a) A suboptimal two stage MUD implementation; (b) MF front end; (c) Projection front end.

Depending on the summary statistic $T^{(k)}(\cdot)$, various structures of user tracker could be formed. In [1], a MF front end is used, as shown in Figure 3(b). The key to this approach is to approximate the MAI of the MF output with Gaussian noise. Note that this is not trivial as the receiver do not have knowledge of the active interfering users at the current slot.
The approach is again, to utilize the Markovian assumption of the channel traffic and adopt the so-called predicted variance for the MF output. Specifically, the output variance of the MF is approximated as

$$
\sigma_{k}^{2}=\sigma^{2}+\sum_{\substack{\gamma_{n-1}^{(j)}=1 \\ j \neq k}}\left(1-q^{(j)}\right) \rho_{k j}^{2} A^{(j)^{2}}+\sum_{\substack{\gamma_{n-1}^{(i)}=0 \\ i \neq k}} p^{(j)} \rho_{k j}^{2} A^{(i)}{ }^{2}
$$

where $\sigma^{2}$ is the channel noise variance, $q^{(j)}$ is the state transition probability from 1 to 0 for user $j$, while $p^{(i)}$ is the state transition probability from 0 to 1 for user $i$. The above Gaussian assumption led to the development of the uniformly most powerful invariant (UMPI) statistic for testing the mean value (zero vs. nonzero) of a Gaussian vector. This statistic follows central or noncentral chi-square distribution depending on which hypothesis is true. The statistic is then fed to the state estimator which also utilize the previous slot's (estimated) state to decide the current status of the particular user.

In this paper, we suggest a different frond end which requires an additional assumption: all users' signature vectors are linearly independent. Specifically, a projection operator is used to mitigate all potential users' interference, as shown in Figure 3(c), where $\mathbf{P}_{\mathbf{C}_{k}}^{\perp}=\mathbf{I}-\mathbf{C}_{k}\left(\mathbf{C}_{k}^{\prime} \mathbf{C}_{k}\right)^{-1} \mathbf{C}_{k}^{\prime}$ and $\mathbf{C}_{k}$ contains spreading vectors of all users' spreading vectors save $\mathbf{c}_{k}$. The input signal is therefore projected onto the orthogonal complement of the space spanned by all other users' signature vectors. Note this front operation is equivalent to a decorrelator assuming full model, hence inevitably enhances the noise variance. The argument is that at the first stage the task is the user identification, not symbol detection, hence the noise enhancement can be tolerated to some extent. The remaining part is similar to the MF front end implementation and a similar UMPI statistic can be developed as the input to the state estimator. The difference, however, is that the projection and matched filter output is precisely Gaussian, hence the input to the state estimate is now exactly calibrated.

Finally, in the absence of traffic information, the UMPI statistic developed in both cases could be used directly to infer about the user status (cf. the dash-dotted curves in Figures 4 and 5). The margin between such one-shot tracking and the approaches using state continuity reveals the gain obtained by using the traffic modeling.

\section{SIMULATION}

Packets were generated for a total number of 20 users, with both on and off periods being geometrically distributed with mean 10 and 100 respectively. Near far effect was considered by placing the user power evenly in the log space with power range of $20 d B$. The SNR shown in the plot was that for the user with minimum power. Packet length was 128 
and the error correction code can correct up to 8 bit errors. The length of the spreading code was 31 .

Figure 4 shows the result of using MF front in the traffic tracking, we note that the one-shot matched filter based tracker does not perform much better than the full model DD. However, when the traffic predictability was used, the HMM based two stage MUD performs almost as well as the true model. This is because the inaccuracy of the Gaussian assumption for the MAI is well compensated via the utilization of the state continuity imposed by the traffic burstiness. Figure 5 is the result of using projection front end, and it is obvious that the performance gain of using traffic is not that significant. Thus in the case of well conditioned input signal, i.e., all $\mathbf{c}_{k}$ 's are linearly independent, the projection front end without using traffic modeling could track the user traffic quite accurately. This linear independence assumption however, puts restriction on the user number as well as the channel condition. If the user number of users exceeds spreading gain, or the channel distortion causes the correlation matrix of $\mathbf{c}_{k}$ 's to lose rank, then the projection front end will fail, at least for those users whose signature vectors lie in the space spanned by other users' signature vectors. A more simple yet robust matched filter front end is favored in these scenarios.

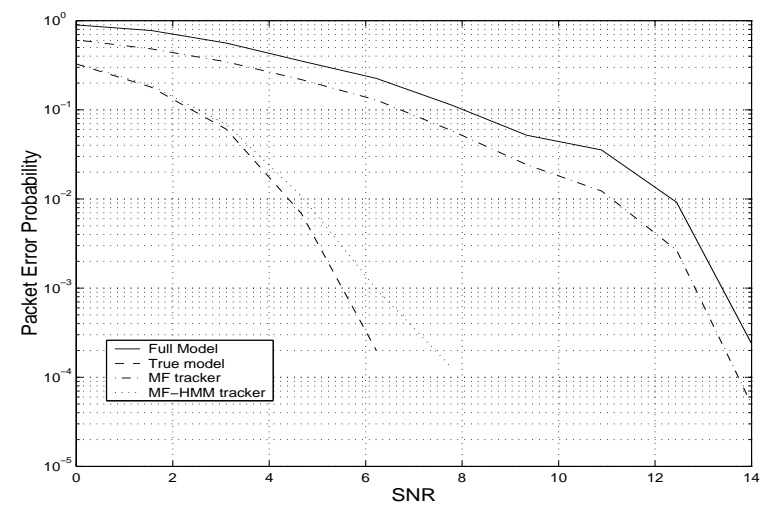

Figure 4: Suboptimal HMM tracker using MF front end.

An important implementation issue is the control of false alarms (an inactive user being claimed active) and miss detections (excluding an active user). A miss detection will result a sure packet loss. Besides, the interference of the missed user on the reception of other active users would not be mitigated at the second stage MUD hence could further hinder the reception performance. Thus the penalty imposed by the miss detection is much more severe than a false alarm. This implies that the minimum error probability state estimate might not yield the best performance in terms of packet reception. A good threshold should be more stringent in controlling the miss detection.

Finally we remark here that the Markovian traffic adopted in this paper is only a first order approximation of the source

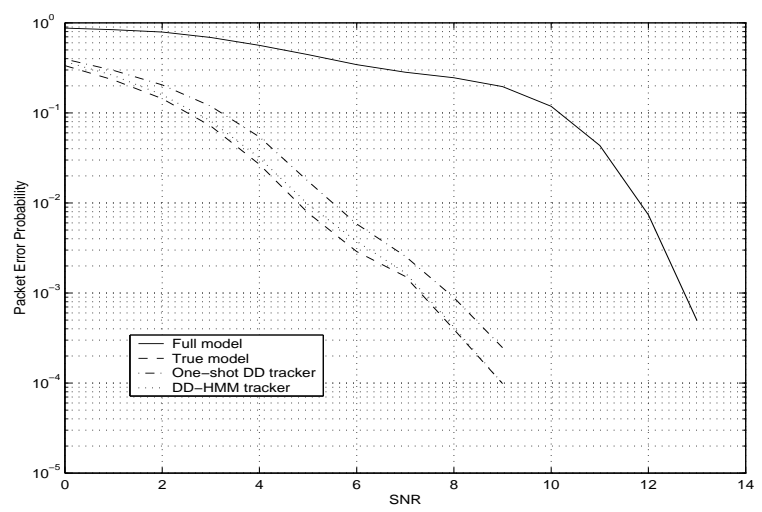

Figure 5: Suboptimal HMM tracker using projection front end.

level traffic. Study of current packet switching network traffic reveals that the packet length as well as the idle period distribution is heavy tailed rather than geometrical, which directly contributes to the so-called self-similarity of network (aggregated) traffic. This heavy-tailedness results in further enhanced predictability of the user traffic at the source level. A more realistic model for such traffic would be higher order Markov chain, though it will inevitably increase the complexity of the user identification procedure.

\section{REFERENCES}

[1] B. Chen and L. Tong. "Traffic aided multiuser detection for packet switching random access/CDMA networks". In Proc. of the 37th Annual Allerton Conference on Communication, Control, and Computing, Monticello, IL, Sep. 1999.

[2] D.R. Cox and D.V. Hinkley. Theoretical Statistics. Chapman and Hall Ltd, New York, 1974.

[3] R.G. Gallager. "Basic limits on protocol information in data communication networks". IEEE Trans. Inform. Theory, IT22:385-399, 1976.

[4] K.W. Halford and M. Brandt-Pearce. "New-User Identification in a CDMA System". IEEE Trans. Communications., 46(1):144-155, January 1998.

[5] U. Mitra and H. V. Poor. "Adaptive decorrelating detectors for CDMA systems". Wireless Person Communications Journal, 2:256-290, Dec. 1995.

[6] L. Rabiner. "A tutorial on hidden Markov Models and selected applications in speech recognition". IEEE Proceedings, 77(2):257-285, Feb. 1989.

[7] D. Raychaudhuri. "Performance analysis of Random Access Packet-Switched Code Division Multiple Access systems". IEEE Trans. Communications, 29(6):895-901, 1981.

[8] A. M. Viterbi. "Approximate analysis of time-synchronous packet networks". IEEE J. Select. Areas Commun., 4:879890, Sep. 1986.

[9] W. Wu and K. Chen. "Identification of active users in synchronous CDMA multiuser detection". IEEE J. Select. Areas Commun., 16(9):1723-1735, Dec. 1998. 